\title{
FUZZY E.O.Q MODEL WITH CONSTANT DEMAND AND SHORTAGES: A FUZZY SIGNOMIAL GEOMETRIC PROGRAMMING (FSGP) APPROACH
}

\author{
Sahidul Islam \\ University of Kalyani, India \\ E-mail: sahidul.math@gmail.com \\ Wasim Akram Mandal \\ University of Kalyani, India \\ E-mail: wasim0018@gmail.com \\ Submission: $24 / 02 / 2017$ \\ Revision: 20/03/2017 \\ Accept: 27/03/2017
}

\section{ABSTRACT}

In this paper, a fuzzy economic order quantity (EOQ) model with shortages under fully backlogging and constant demand is formulated and solved. Here the model is solved by fuzzy signomial geometric programming (FSGP) technique. Fuzzy signomial geometric programming (FSGP) technique provides a powerful technique for solving many non-linear problems. Here we have proposed a new idea that is fuzzy modified signomial geometric programming (FMSGP) and some necessary theorems have been derived. Finally, these are illustrated by some numerical examples and applications.

Keywords: EOQ model, Nearest Interval Approximation (NIA), Fuzzy number, Signomial Geometric Programming.

Mathematics Subject Classification: 90B05, 90C70. 
DOI: 10.14807/ijmp.v8i4.640

\section{INTRODUCTION}

An inventory management deals with the decision that minimizes total averages cost or maximizes total average profit. In an ordinary inventory model are considered all parameters like shortage cost, carrying cost etc. as a fixed, but in a real life situation there some small fluctuations. Therefore, consideration of fuzzy number is more realistic and interesting.

The study of inventory model where demand rates vary with time is the last decades. Datta and Pal investigated an inventory system with power demand pattern and deterioration. Park and Wang studied shortages and partial backlogging of items. Friedman (1978) presented continuous time inventory model with time varying demand.

Ritchie (1984) studied an inventory model with linear increasing demand. Goswami and Chaudhuri (1991) discussed an inventory model with shortages. Gen et. al. (1997) considered classical inventory model with triangular fuzzy number. Yao and Lee (1998) considered an economic production quantity model in fuzzy sense. De, Kundu and Goswami (2003) presented an economic production quantity inventory model involving fuzzy demand rate.

Syde and Aziz (2007) applied sign distance method to fuzzy inventory model without shortage. D.Datta and Pravin Kumar published several paper of fuzzy inventory with or without shortage. Islam, Roy (2006) presented a fuzzy EPQ model with flexibility and reliability consideration and demand depended unit production cost under a space constraint.

A solution method of posynomial geometric programming with interval exponents and coefficients was developed by Liu (2008). Kotba. M. Kotb, Halla. Fergancy (2011) presented Multi-item EOQ model with both demand-depended unit cost and varying lead time via geometric programming.

Jana, Das and Maiti (2014) presented multi-item partial backlogging inventory models over random planning horizon in random fuzzy environment. Samir Dey and Tapan Kumar Roy (2015) presented optimum shape design of structural model with imprecise coefficient by parametric geometric programming. 
A signomial optimization problem often provides much more accurate mathematical representation of real-world nonlinear optimization problems. Initially Passy and Wilde (1967), and Blau and Wilde (1969) generalized some of the prototype concepts and theorems in order to treat signomial programs (SP).

In other work that general type of signomial programming (SP) has been done by Charnes et. al. (1988), who proposed methods for approximating signomial programs with prototype geometric programs. Islam and Roy (2005) proposed EOQ model with shortages under fully backlogging and constant demand is formulated and solved. Here the model is solved by fuzzy signomial geometric programming (FSGP) technique. Fuzzy signomial geometric programming (FSGP) technique provides a powerful technique for solving many non-linear problems.

\section{FUZZY NUMBER AND ITS NEAREST INTERVAL APPROXIMATION}

\subsection{Fuzzy number}

A real number $\tilde{A}$ described as fuzzy subset on the real line ${ }^{\mathcal{R}}$ whose membership function $\mu_{\tilde{A}}(x)$ has the following characteristics with $-\propto<a_{1} \leq a_{2} \leq a_{3}<\propto$

$\mu_{\tilde{A}}(x)= \begin{cases}\mu_{\tilde{A}}^{L}(x) & \text { if } a_{1} \leq x \leq a_{2}, \\ \mu_{\tilde{A}}^{R}(x) & \text { if } a_{2} \leq x \leq a_{3}, \\ 0 & \text { otherwise. }\end{cases}$

Where $\mu_{A}^{L}(x):\left[a_{1}, a_{2}\right] \rightarrow[0,1]$ is continuous and strictly increasing and $\mu_{\tilde{A}}^{R}(x):\left[a_{2}, a_{3}\right] \rightarrow[0,1]$ is continuous and strictly decreasing.

$\alpha$ - level set: The $\alpha$ - level of a fuzzy number is defined as a crisp set where $A(\alpha)=[x: \mu A(x) \geq \alpha, x \in X]$ where $\alpha \in[0,1] . A(\alpha)$ is a non-empty bounded closed interval contained in $X$ and it can be denoted by $\operatorname{A\alpha }=[\operatorname{AL}(\alpha), \operatorname{AR}(\alpha)] . \operatorname{AL}(\alpha)$ and $\operatorname{AR}(\alpha)$ are the lower and the upper bounds of the closed interval, respectively.

\subsection{Interval number}

An interval number $A$ is defined by an ordered pair of real numbers as follows $\left.\mathrm{A}=\left[a_{L}, a_{R}\right]=\left\{x: a_{L} \leq x \leq a_{R}\right\}, x \in \mathcal{R}\right\}$ where $a_{L}$ and $a_{R}$ are the left and the right bounds of interval $A$, respectively. The interval $A$, is also defined by center $\left({ }^{a_{c}}\right)$ and half-width $\left(a_{w}\right)$ as follows 
$\mathrm{A}=\left(a_{c}, a_{W}\right)=\left\{\mathrm{x}: a_{c}-a_{w} \leq x \leq a_{c}+a_{w}, x \in \mathcal{R}\right\}$ where $a_{c}=\frac{\frac{a_{R}+a_{L}}{2}}{2}$ is the center and $a_{w}$ $=\frac{\frac{a_{R}-a_{L}}{2}}{2}$ is the half-width of $A$.

\subsection{Nearest interval approximation}

Here we want to approximate a fuzzy number by a crisp model. Suppose $\tilde{A}$ and $^{\tilde{B}}$ are two fuzzy numbers with $\alpha$-cuts are $[A L(\alpha), A R(\alpha)]$ and $[B L(\alpha), B R(\alpha)]$, respectively. Then the distance between $\tilde{A}$ and $\tilde{B}^{\tilde{B}}$ is

$$
\mathrm{d}(\tilde{A}, \tilde{B})=\sqrt{\int_{0}^{1}\left(\mathrm{~A}_{\mathrm{L}}(\alpha)-\mathrm{B}_{\mathrm{L}}(\alpha)\right)^{2}+\int_{0}^{1}\left(A_{R}(\alpha)-B_{R}(\alpha)\right)^{2} d \alpha} .
$$

Given ${ }^{a}$ fuzzy number $\hat{A}$, We have to find a closed interval $C_{D}(\tilde{A})$, which is closest to $\hat{A}^{\widehat{A}}$ with respect to some metric. We can do it, since each interval is also a fuzzy number with constant $\alpha$-cut for all $\alpha \in[0,1]$. Hence $\left(C_{D}(\tilde{A})\right) \alpha=\left[C_{L}, C_{R}\right]$. Now we have to minimize

$$
\mathrm{d}\left(\tilde{A}, C_{D}(\tilde{A})\right)=\sqrt{\int_{0}^{1}\left(\mathrm{~A}_{\mathrm{L}}(\alpha)-C_{L}\right)^{2}+\int_{0}^{1}\left(A_{R}(\alpha)-C_{R}\right)^{2} d \alpha}
$$

with respect to $C_{L}$ and $C_{R}$.

In order to minimize $\mathrm{d}\left(\tilde{A}, C_{D}(\tilde{A})\right)$, it is sufficient to minimize the function $\mathrm{D}\left(C_{L}, C_{R}\right)=\left(d^{2}\left(\tilde{A}, C_{D}(\tilde{A})\right)\right)$. The first partial derivatives are

$\frac{\partial}{\partial c_{L}} \mathrm{D}\left(C_{L}, C_{R}\right)=-2 \int_{0}^{1} \mathrm{~A}_{\mathrm{L}}(\alpha) \mathrm{d} \alpha+2 C_{L}$ and $\frac{\partial}{\partial c_{R}} \mathrm{D}\left(C_{L^{\prime}} C_{R}\right)=-2 \int_{0}^{1} \mathrm{~A}_{\mathrm{R}}(\alpha) \mathrm{d} \alpha+2 C_{R^{*}}$.

Solving $\frac{\partial}{\partial c_{L}} \mathrm{D}\left(C_{L}, C_{R}\right)=0$ and $\frac{\partial}{\partial c_{R}} \mathrm{D}\left(C_{L}, C_{R}\right)=0$, we get $\mathrm{CL}=\int_{0}^{1} A_{L}(\alpha) d \alpha$ and $\mathrm{CR}=$ $\int_{0}^{1} A_{R}(\alpha) d \alpha$.

Again, since $\frac{\partial^{\mathrm{a}}}{\partial C_{L}{ }^{\mathrm{a}}}\left(\mathrm{D}\left(C_{L}^{*}, C_{R}^{*}\right)\right)=2>0, \frac{\partial^{\mathrm{a}}}{\partial C_{R}{ }^{2}}\left(\mathrm{D}\left(C_{L}^{*}, C_{R}^{*}\right)\right)=2>0$ and $\left.\mathrm{H}\left(C_{L}^{*}, C_{R}^{*}\right)=\frac{\partial^{\mathrm{a}}}{\partial C_{L}{ }^{\mathrm{a}}}\left(\mathrm{D}\left(C_{L}^{*}, C_{R}^{*}\right)\right)\right)^{\frac{\partial^{\mathrm{a}}}{\partial C_{R}{ }^{\mathrm{a}}}}\left(\mathrm{D}\left(C_{L}{ }^{*}, C_{R}{ }^{*}\right)\right)-\left(\frac{\partial^{\mathrm{a}}}{\partial C_{L}{ }^{*} C_{R}{ }^{*}}\left(\mathrm{D}\left(C_{L}{ }^{*}, C_{R}{ }^{*}\right)\right)\right)^{2}=4>0$.

So, $\mathrm{D}\left(C_{L}{ }^{*}, C_{R}{ }^{*}\right)$ i.e. $\mathrm{d}\left(\tilde{A}, C_{D}(\tilde{A})\right)$ is global minimum. Therefore, the interval $\mathrm{Cd}(\tilde{A})=$ $\left[\int_{0}^{1} A_{L}(\alpha) d \alpha, \int_{0}^{1} A_{R}(\alpha) d \alpha\right]$ is the nearest interval approximation of fuzzy number $\tilde{A}$ with respect to the metric $d$. 
Let $\tilde{A}=($ a1, a2, a3 $)$ be a triangular fuzzy number. The a-cut interval of $\tilde{A}$ is defined as

$A \alpha=\left[A_{L}(\alpha), A_{R}(\alpha)\right]$ where $A_{L}(\alpha)=a 1+\alpha(a 2-a 1)$ and $A_{R}(\alpha)=a 3-\alpha(a 3-a 2)$. By nearest interval approximation method the lower limit of the interval is

$\mathrm{CL}=\int_{0}^{1} A_{\mathrm{L}}(\alpha) d \alpha=\int_{0}^{1}\left[\mathrm{a}_{1}+\alpha\left(\mathrm{a}_{2}-\mathrm{a}_{1}\right)\right] \mathrm{d} \alpha=\frac{\mathrm{a}_{1}+\mathrm{a}_{\mathrm{g}}}{2}$ and the upper limit of the interval is $\mathrm{CR}=\int_{0}^{1} A_{R}(\alpha) d \alpha=\int_{0}^{1}\left[\mathrm{a}_{3}-\alpha\left(\mathrm{a}_{3}-\mathrm{a}_{2}\right)\right] \mathrm{d} \alpha=\frac{\mathrm{a}_{3}+\mathrm{a}_{3}}{2}$.

Therefore, the interval number corresponding $\tilde{A}$ is $\left[\frac{a_{1}+a_{n}}{2}, \frac{a_{8}+a_{n}}{2}\right]=[m, n]$. In the centre and half -width form the interval number of ${ }^{\tilde{A}}$ is defined as $\left\langle\frac{1}{4}\left(\mathrm{a}_{1}+2 \mathrm{a}_{2}+\mathrm{a}_{3}\right)_{,} \frac{1}{4}\left(\mathrm{a}_{3}-\mathrm{a}_{1}\right)\right\rangle$.

\subsection{Parametric Interval-valued function}

Let $[\mathrm{m}, \mathrm{n}]$ be an interval, where $\mathrm{m}>0, \mathrm{n}>0$. From analytical geometry point of view, any real number can be represented on a line. Similarly, we can express an interval by a function. The parametric interval-valued function for the interval $[\mathrm{m}, \mathrm{n}]$ can be taken as $\mathrm{g}(\mathrm{s})=m^{1-s} n^{s}$ for $\mathrm{s} \in[0,1]$, which is a strictly monotone, continuous function and its inverse exits. Let ${ }^{\psi}$ be the inverse of $g(s)$, then

$$
\mathrm{S}=\frac{\log \psi-\log m}{\log n-\log m} .
$$

\section{DETERMINISTIC EOQ MODEL}

In many real-life situations shortages occur in an EOQ model. When Shortages occurs, costs are incurred. The purpose of this section is to discuss the deterministic EOQ model in crisp environment. The notations to be used are:

Tac(Q,S): Total average cost of the EOQ model.

Q: Order quantity.

$Q-S:$ Maximum shortage that occurs under an ordering policy

$c_{s:}$ Carrying cost per item per unit time.

$c_{h}$ : Shortages cost per item per unit time.

$c_{0}$ : Ordering cost per order. 
ISSN: 2236-269X

DOI: 10.14807/ijmp.v8i4.640

D: Demand rate per unit time.

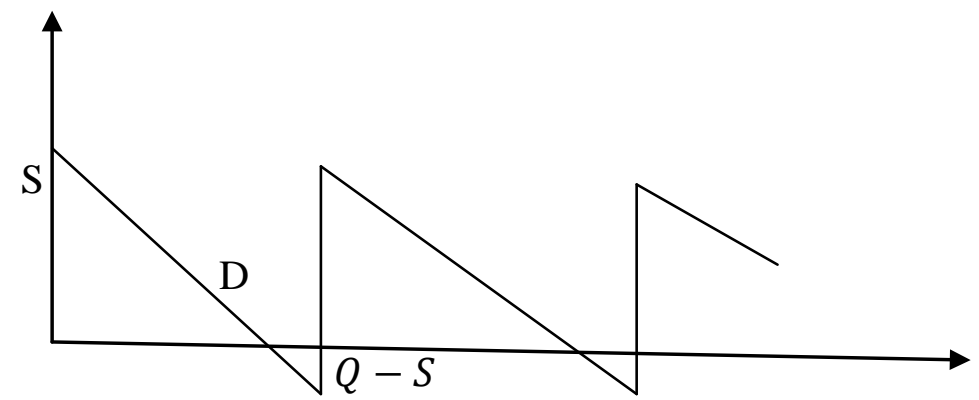

0

$\mathrm{t}$

Figure 2: EOQ model

Variables of the EOQ model are Q, S and $c_{0}, c_{h}, c_{s}$ are constant parameters.

Thus,

Total carrying cost $=\frac{\varepsilon_{s} s^{2}}{2 D}$,

Total shortages cost $=\frac{\sigma_{h}(q-s)^{2}}{2 D}$,

So total cost $=c_{0}+\frac{c_{h}(q-s)^{2}}{2 D}+\frac{c_{s} s^{2}}{2 D}$

And total average cost $\operatorname{Tac}(\mathrm{Q}, \mathrm{S})=\frac{1}{t}\left[c_{0}+\frac{\sigma_{h}(q-s)^{2}}{2 D}+\frac{\epsilon_{g} S^{2}}{2 D}\right]$

$$
=\frac{C_{0} \mathrm{D}}{Q}+\frac{C_{h}(\mathrm{Q}-\mathrm{S})^{2}}{2 Q}+\frac{C_{s}(\mathrm{~S})^{2}}{2 Q}, \quad\left[t=\frac{Q}{D}\right] .
$$

i.e., problem is

Minimize $\operatorname{Tac}(\mathrm{Q}, \mathrm{S})=\frac{c_{0} \mathrm{D}}{Q}+\frac{c_{h}(\mathrm{Q}-\mathrm{S})^{2}}{2 Q}+\frac{c_{s}(\mathrm{~S})^{2}}{2 Q}$

subject to $\mathrm{Q}, \mathrm{S}>0$.

\section{FUZZY EOQ MODEL}

In the inventory model we take the parameters $\widetilde{c}_{0}, \widetilde{c}_{h}$ and $\widetilde{c}_{s}$ are fuzzy numbers.

Then from (3.1) we have 


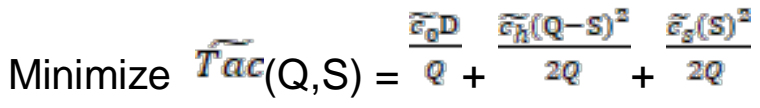

subject to $Q, S>0$.

\section{UNCONSTRAINED FUZZY SIGNOMIAL GP PROBLEM}

A problem without any restrictions is called unconstrained problem. I.e., a problem of the form

Minimize $g_{0}\left(x_{1}, x_{2}, \ldots \ldots \ldots, x_{m}\right)$

Subject to $x_{j}>0, \mathrm{j}=1,2, \ldots \ldots, \mathrm{m}$,

is called unconstrained problem.

\section{Primal problem:}

A primal fuzzy signomial GP programming problem is of the form

Minimize $\tilde{g}_{0}\left(x_{1}, x_{2}, \ldots \ldots \ldots, x_{m}\right)$

Subject to $x_{j}>0, \mathrm{j}=1,2, \ldots \ldots, \mathrm{m}$.

Where $\tilde{g}_{0}(x)=\sum_{i=1}^{k} \sigma_{i} \tilde{c}_{i} \prod_{j=1}^{m} x_{j}{ }^{a_{i j}}$.

Here $a_{i j}$ are real numbers and coefficient $\tilde{c}_{i}$ are fuzzy triangular, as $\tilde{c}_{i}=\left(c_{i}{ }^{1}, c_{i}{ }^{2}, c_{i}{ }^{3}\right)$.

Using nearest interval approximation method, transformed all triangular fuzzy number into interval number i.e., $\left[c_{i}{ }^{L}, c_{i}{ }^{U}\right]$. Then the fuzzy signomial geometric programming problem is of the following form

$\operatorname{Min} \hat{g}_{0}(x)=\sum_{i=1}^{k} \sigma_{i} \hat{c}_{i} \prod_{j=1}^{m} x_{j} a_{i j}$

Subject to $\quad x_{j}>0, \mathrm{j}=1,2, \ldots \ldots, \mathrm{m}$.

Where $\hat{c}_{i}$ denotes the interval counter parts i.e., $\hat{c}_{i} \in\left[c_{i}{ }^{L}, c_{i}{ }^{U}\right] \cdot c_{i}{ }^{L}>0, c_{i}{ }^{U}>0$, for all i. Using parametric interval-valued functional form, the problem () reduces to Min $g_{0}(x, s)=\sum_{i=1}^{k} \sigma_{i}\left(c_{i}^{L}\right)^{1-s}\left(c_{i}^{U}\right)^{s} \prod_{j=1}^{m} x_{j}^{a_{i j}}$

Subject to $\quad x_{j}>0, \mathrm{j}=1,2, \ldots \ldots, \mathrm{m}$. 
This is a parametric geometric programming (PGP) problem.

\section{Dual signomial GP problem:}

Dual GP problem of the given primal GP problem is

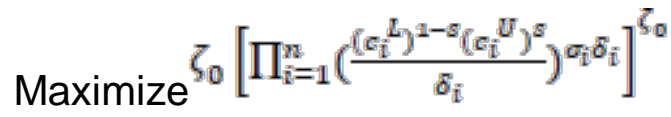

Subject to $\sum_{i=1}^{k} \sigma_{i} \delta_{i}=\zeta_{0}$,

$$
\begin{aligned}
& \sum_{i=1}^{k} \sigma_{i} a_{i j} \delta_{i}=0, \quad j=1,2, \ldots \ldots \ldots, m \\
& \delta_{i}>0,
\end{aligned}
$$

Case I: $n>m+1$, (i.e. $D D>0$ ) so the DP presents a system of linear equations for the dual variables. Here the number of linear equations is less than the number of dual variables. More solutions of dual variable vector exist. In order to find an optimal solution of DP, we need to use some algorithmic methods.

Case II: $n<m+1$, (i.e. $D D<0$ ) so the DP presents a system of linear equations for the dual variables. Here the number of linear equations is greater than the number of dual variables. In this case generally no solution vector exists for the dual variables. However, using Least Square (LS) or Min-Max (MM) method one can get an approximate solution for this system.

Furthermore the primal-dual relation is

$\left(c_{i}{ }^{L}\right)^{1-s}\left(c_{i}{ }^{U}\right)^{s} \prod_{j=1}^{m} x_{j}^{*} a_{i j}=\zeta_{0} \delta^{*}{ }_{i} v\left(\delta^{*}, s^{*}\right)$.

Note: A Weak Duality theorem would say that

$$
g_{0}(x, s) \geq v(\delta, s)
$$

For any primal-feasible $\mathrm{x}$ and dual-feasible $\delta$ but this is not true of the pseudodual fuzzy signomial GP problem.

Corollary: When the value of $\sigma_{\bar{i}}$ is 1 , then a fuzzy signomial geometric programming (FSGP) problem transform to ordinary geometric programming problem.

Theorem 1: When $\sigma_{i}$ is 1 , then $g_{o}(\mathrm{x}, \mathrm{s}) \geq v(\delta, \mathrm{s})$ (Primal- Dual Inequality).

\section{Proof}

The expression for $g_{0}(x, s)$ can be written as 
ISSN: 2236-269X

DOI: $10.14807 /$ ijmp. v8i4.640

$g_{o}(\mathrm{x}, \mathrm{s})=\sum_{i=1}^{n} \delta_{k}\left(\frac{\left(c_{i}^{L}\right)^{1-s_{(}}\left(c_{i}^{U}\right)^{s} \Pi_{j=1}^{m} x_{j}^{\alpha_{k j}}}{\delta_{k}}\right)$.

Here the weights are $\delta_{1}, \delta_{2}, \ldots \ldots, \delta_{n}$ and positive terms are $\frac{\left(c_{1}\right)^{1-s_{(}}\left(c_{1}\right)^{U} \Pi_{j=1}^{m} x_{j}^{\alpha_{1 j}}}{\delta_{1}}$,

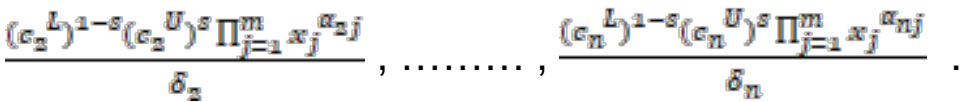

Now applying A.M.-.G.M inequality, we get

$\left(\frac{\left(c_{1}{ }^{L}\right)^{1-s}\left(c_{1}{ }^{U}\right)^{s} \prod_{j=1}^{m} x_{j}^{\alpha_{1 j}}+\left(c_{2}{ }^{L}\right)^{1-s}\left(c_{2}{ }^{U}\right)^{s} \prod_{j=1}^{m} x_{j}^{\alpha_{n j}}+\ldots+\left(c_{n}{ }^{L}\right)^{1-s}\left(c_{n}{ }^{U}\right)^{s} \prod_{j=1}^{m} x_{j}^{\alpha_{n j}}}{\left(\delta_{1}+\delta_{2}+\cdots+\delta_{n}\right)}\right)^{\left(\delta_{01}+\delta_{0 s}+\cdots+\delta_{n}\right)}$

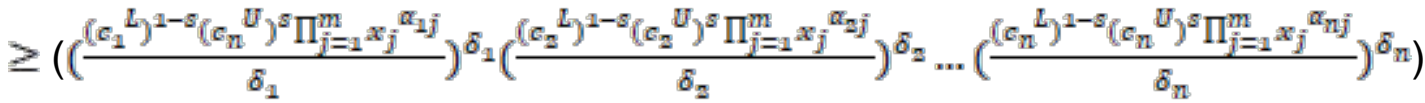

Or $\quad\left(\frac{g_{0}(x, s)}{\sum_{i=1}^{n} \delta_{i}}\right)^{\sum_{i=1}^{n} \delta_{i}} \geq \prod_{i=1}^{n}\left(\frac{\left.\left(c_{i}^{L}\right)^{1-s}\left(c_{i}\right)_{i}\right)^{s} \prod_{j=1}^{m} x_{j}^{\alpha_{n j}}}{\delta_{i}}\right)^{\delta_{i}} \quad$ [as $\left.\sum_{i=1}^{n} \delta_{k}=1\right]$

Or $\quad g_{0}(\mathrm{x}, \mathrm{s}) \geq\left(\frac{\left(c_{i}^{L}\right)^{\left.1-s_{(}\left(_{i}\right)^{U}\right)^{s}}}{\delta_{k}}\right)^{\sum_{i=1}^{n} \delta_{i}} \prod_{j=1}^{m} x_{j}^{\sum_{i=1}^{n} \alpha_{i j} \delta_{i}}$

Or

$$
\begin{aligned}
& g_{0}(\mathrm{x}, \mathrm{s}) \geq \prod_{i=1}^{n}\left(\frac{\left(c_{i}^{L}\right)^{\left.1-s_{\left(c_{i}\right.}\right)^{s}}}{\delta_{i}^{s}}\right)^{\delta_{i}} \prod_{j=1}^{m} x_{j}^{\sum_{i=1}^{n} \alpha_{i j} \delta_{i}} \\
& =\prod_{i=1}^{n}\left(\frac{\left(c_{i}^{L}\right)^{\left.1-\delta_{(}\left(_{i}\right)^{U}\right)^{s}}}{\delta_{i}}\right)^{\delta_{i}}=v(\delta, \mathrm{s}) \\
& {\left[a s \sum_{k=1}^{T_{0}} \alpha_{0 k j} \delta_{o k}=0\right]}
\end{aligned}
$$

i.e., $\quad g_{0}(\mathrm{X}, \mathrm{s}) \geq v(\delta, \mathrm{s})$.

Ex. 1: Minimize $T \widetilde{T a c}(Q, S)=\frac{\widetilde{F}_{0} \mathrm{D}}{Q}+\frac{\widetilde{h}_{h}(Q-S)^{2}}{2 Q}+\frac{\widetilde{q}_{s}(S)^{2}}{2 Q}$

subject to $Q, S>0$.

With input values

Table-1 (Input data)

\begin{tabular}{|c|c|c|c|}
\hline$\widetilde{\mathrm{c}_{0}}=\widetilde{20}$ & $\widetilde{\mathrm{c}_{\mathrm{h}}}=\widetilde{50}$ & $\widetilde{\mathrm{c}_{s}}=\widetilde{50}$ & $\mathrm{D}$ \\
\hline$(16,20,24)$ & $(40,50,60)$ & $(40,50,60)$ & 10 \\
\hline
\end{tabular}

Using nearest approximation method

$\widetilde{20}=(16,20,24) \approx[18,22] \approx 18^{1-s} 22^{s} \in[18,22] ;$

$\widetilde{50}=(40,50,60) \approx[45,55] \approx 45^{1-s} 55^{s} \in[45,55] ; \mathrm{s} \in[0,1]$.

Then the problem is 
DOI: 10.14807/ijmp.v8i4.640

Min. $\operatorname{Tac}(\mathrm{Q}, \mathrm{S}, \mathrm{S})=\frac{18^{1-s_{2}} \cdot 2^{s} \cdot 10}{Q}+\frac{45^{1-s_{5}} 5^{s}(\mathrm{Q}-\mathrm{S})^{2}}{2 Q}+\frac{45^{1-s^{s}} 55^{s}(\mathrm{~S})^{2}}{2 Q}$

Sub. Q, $\mathrm{S}>0$.

i.e., $\quad \operatorname{Min}$. $\operatorname{Tac}(\mathrm{Q}, \mathrm{S}, \mathrm{S})=\frac{18^{1-s_{2}} 22^{s} \cdot 10}{Q}+\frac{45^{1-s_{5}} 55^{s}(\mathrm{~S})^{2}}{Q}+\frac{45^{1-s} 55^{s} Q}{2}-\frac{45^{1-s_{5}} 55^{s} S}{1}$

Sub. $\mathrm{Q}, \mathrm{S}>0$.

This is primal problem and corresponding dual problem is

$v(\delta, s)=\left(\frac{10.16^{1-s_{2}} 20^{s}}{\delta_{1}}\right)^{\delta_{1}}\left(\frac{45^{1-s} 55^{s}}{\delta_{2}}\right)^{\delta_{z}}\left(\frac{45^{1-s} 55^{s}}{2 \delta_{3}}\right)^{\delta_{a}}\left(\frac{45^{1-s} 55^{s}}{\delta_{4}}\right)^{-\delta_{4}}$

Subject to

$$
\begin{aligned}
& \delta_{1}+\delta_{2}+\delta_{3}-\delta_{4}=1, \\
& -\delta_{1}-\delta_{2}+\delta_{3}=0, \\
& 2 \delta_{2}-\delta_{4}=0,
\end{aligned}
$$

Solving above equations, we have

$$
\begin{aligned}
& \delta_{4}=2 \delta_{2}, \quad \delta_{3}=1-\delta_{1}-\delta_{2}+\delta_{4}=1-\delta_{1}-\delta_{2}+2 \delta_{2}=1-\delta_{1}+\delta_{2}, \\
& \delta_{1}+\delta_{2}=1-\delta_{1}+\delta_{2}, \delta_{1}=\frac{1}{2}, \delta_{3}=\frac{1}{2}+\delta_{2} . \\
& \text { i.e., } v(\delta, s)=\left(\frac{10.16^{1-s_{24}}}{1 / 2}\right)^{1 / 2}\left(\frac{45^{1-s_{55}}}{\delta_{2}}\right)^{\delta_{2}}\left(\frac{45^{1-s_{55}}}{2\left(0.5+\delta_{3}\right)}\right)^{\left(0.5+\delta_{2}\right)}\left(\frac{45^{1-s_{55}}}{2 \delta_{2}}\right)^{-2 \delta_{2}}
\end{aligned}
$$

Taking log on both side of (5.7) and then partially differentiating with respect to $\delta_{2}$ and using the conditions of finding optimal solution we get this equation

$$
\begin{aligned}
& \left(\log 2.45^{1-s} 55^{s}-\log 2 \delta_{2}\right)+\left(\log 45^{1-s} 55^{s}-\log 2\left(0.5+\delta_{2}\right)-2\left(\log 45^{1-s} 55^{s}\right.\right. \\
& \left.-\log 2 \delta_{2}\right)=0 \\
& \Rightarrow \log \left(2.45^{1-s} 55^{s}\right) / 45^{1-s} 55^{s}-\log 2 \delta_{2}\left(1+2 \delta_{2}\right)=0 \\
& \Rightarrow \delta_{2}\left(1+2 \delta_{2}\right)=1 \text {. }
\end{aligned}
$$

From primal-dual relation

$$
\begin{aligned}
& \frac{10.16^{1-s} 24^{s}}{Q}=\delta_{1} v(\delta), \\
& \frac{45^{1-s} 55^{s} s^{2}}{2 Q}=\delta_{2} v(\delta),
\end{aligned}
$$


ISSN: 2236-269X

DOI: 10.14807/ijmp.v8i4.640

$$
\begin{aligned}
& \frac{45^{1-s} 55^{s} Q}{2}=\delta_{3} v(\delta), \\
& 45^{1-s} 55^{s} S=\delta_{4} v(\delta) .
\end{aligned}
$$

Solving above relations with difference values of weight, we get the list of values in table-2

\begin{tabular}{|c|c|c|c|c|c|}
\hline \multirow[b]{2}{*}{$\mathrm{s}$} & \multirow[b]{2}{*}{$1-s$} & \multirow[b]{2}{*}{ Optimal dual variables } & \multirow[b]{2}{*}{$\begin{array}{l}\text { Optimal primal } \\
\text { variables }\end{array}$} & \multicolumn{2}{|c|}{ Optimal values objectives } \\
\hline & & & & $\mathrm{v}(\delta)$ & $\operatorname{Tac}(\mathrm{Q} S)$ \\
\hline 0.1 & 0.9 & $\begin{array}{l}\delta_{1}^{*}=0.5 \\
\delta_{2}^{*}=0.5 \\
\delta_{3}^{*}=1, \delta_{4}^{*}=1 .\end{array}$ & $\begin{array}{l}\mathrm{S}^{*}=1.905 \\
\mathrm{Q}^{*}=3.810\end{array}$ & 87.464 & 87.464 \\
\hline 0.3 & 0.7 & $\begin{array}{l}\delta_{1}^{*}=0.5 \\
\delta_{2}^{*}=0.5 \\
\delta_{3}^{*}=1, \delta_{4}^{*}=1 .\end{array}$ & $\begin{array}{l}\mathrm{S}^{*}=1.944 \\
\mathrm{Q}^{*}=3.889\end{array}$ & 92.929 & 92.929 \\
\hline 0.5 & 0.5 & $\begin{array}{l}\delta_{1}^{*}=0.5 \\
\delta_{2}^{*}=0.5 \\
\delta_{3}^{*}=1, \delta_{4}^{*}=1 .\end{array}$ & $\begin{array}{l}\mathrm{S}^{*}=1.984 \\
\mathrm{Q}^{*}=3.969\end{array}$ & 98.736 & 98.736 \\
\hline 0.7 & 0.3 & $\begin{array}{l}\delta_{1}^{*}=0.5 \\
\delta_{2}^{*}=0.5 \\
\delta_{3}^{*}=1, \delta_{4}^{*}=1 .\end{array}$ & $\begin{array}{l}\mathrm{S}^{*}=2.026 \\
\mathrm{Q}^{*}=4.051\end{array}$ & 104.906 & 104.906 \\
\hline 0.9 & 0.1 & $\begin{array}{l}\delta_{1}^{*}=0.5 \\
\delta_{2}^{*}=0.5 \\
\delta_{3}^{*}=1, \delta_{4}^{*}=1 .\end{array}$ & $\begin{array}{l}\mathrm{S}^{*}=2.068 \\
\mathrm{Q}^{*}=4.135\end{array}$ & 111.462 & 111.462 \\
\hline
\end{tabular}

Table -2: optimal solution

\section{FUZZY MODIFIED SIGNOMIAL GEOMETRIC PROGRAMMING PROBLEM (FMSGP)}

\subsection{Primal problem:}

A primal modified signomial GP programming problem is of the form

Minimize $\tilde{g}_{0}\left(x_{l j}\right)$

Subject to $\quad x_{l j}>0, \mathrm{j}=1,2, \ldots \ldots, \mathrm{m}$.

Where $\tilde{g}_{0}(x)=\sum_{l=1}^{n} \sum_{i=1}^{k} \sigma_{l i} \tilde{c}_{l i} \prod_{j=1}^{m} x_{l j}{ }^{a_{l i j}}$.

Using nearest interval approximation method, transformed all triangular fuzzy number into interval number i.e., $\left[c_{l i}{ }^{L}, c_{l i}{ }^{U}\right]$. Then the fuzzy signomial geometric programming problem is of the following form

Minimize $\hat{g}_{0}\left(x_{1}, x_{2}, \ldots \ldots \ldots, x_{m}\right)$ 
Subject to $x_{j}>0, \mathrm{j}=1,2, \ldots \ldots, \mathrm{m}$.

Where $\hat{g}_{0}(x)=\sum_{l=1}^{n} \sum_{i=1}^{k} \sigma_{l i} \hat{c}_{l i} \prod_{j=1}^{m} x_{j}^{a_{l i j}}$.

Where $\hat{c}_{l i}$ denotes the interval counter parts i.e., $\hat{c}_{l i} \in\left[c_{l i}{ }^{L}, c_{l i}{ }^{U}\right] \cdot c_{l i}{ }^{L}>0, c_{l i}{ }^{U}>0$, for all i. Using parametric interval-valued functional form, the problem () reduces to

Minimize $g_{0}\left(x_{1}, x_{2}, \ldots \ldots \ldots, x_{m}, s\right)$

Subject to $\quad x_{j}>0, \mathrm{j}=1,2, \ldots \ldots, \mathrm{m}$.

Where $g_{0}(x, s)=\sum_{l=1}^{n} \sum_{i=1}^{k} \sigma_{l i}\left(c_{l i}{ }^{L}\right)^{1-s}\left(c_{l i}{ }^{U}\right)^{s} \prod_{j=1}^{m} x_{j}{ }^{a_{l i j}}$.

This is a parametric geometric programming (PGP) problem.

\section{Dual signomial GP problem:}

Dual GP problem of the given primal GP problem is

Maximize $\zeta_{0}\left[\prod_{l=1}^{n} \prod_{i=1}^{k}\left(\frac{\left(\sigma_{l i}^{L}\right)^{\left.1-s_{\left(\sigma_{l i}\right.}{ }^{U}\right)^{s}}}{\delta_{l i}}\right)^{\sigma_{l i} \delta_{l i}}\right]^{\zeta_{0}}$

Subject to $\sum_{i=1}^{n} \sum_{i=1}^{k} \sigma_{l i} \delta_{l i}=\zeta_{0}$,

$$
\begin{aligned}
& \sum_{l=1}^{n} \sum_{i=1}^{k} \sigma_{l i} a_{l i j} \delta_{l i}=0, \quad j=1,2, \ldots \ldots \ldots, m . \\
& \delta_{l i}>0,
\end{aligned}
$$

Case I: $n k \geq n m+n$, (i.e. $D D>0$ ) So the DP presents a system of linear equations for the dual variables. Here the number of linear equations is less than the number of dual variables. More solutions of dual variable vector exist. In order to find an optimal solution of DP, we need to use some algorithmic methods.

Case II: $n k<n m+n$, (i.e. DD <0) So the DP presents a system of linear equations for the dual variables. Here the number of linear equations is greater than the number of dual variables. In this case generally no solution vector exists for the dual variables. However, using Least Square (LS) or Min-Max (MM) method one can get an approximate solution for this system.

Furthermore the primal-dual relation is 
INDEPENDENT JOURNAL OF MANAGEMENT \& PRODUCTION (IJM\&P)

http://www.ijmp.jor.br

v. 8 , n. 4, October - December 2017

ISSN: 2236-269X

DOI: 10.14807/ijmp.v8i4.640

$\left(c_{l i}{ }^{L}\right)^{1-s}\left(c_{l i}{ }^{U}\right)^{s} \prod_{j=1}^{m} x_{l j}{ }^{a_{l i j}}=\zeta_{0} \delta^{*}{ }_{l i} \sqrt[n]{v\left(\delta^{*}\right)},(l=1,2, \ldots \ldots, k ; i=1,2, \ldots \ldots \ldots, n)$,

$\mathrm{SE}[0,1] . \quad(6.5)$

Note 2: A Weak Duality theorem would say that

$g_{0}\left(x_{l j}, s\right) \geq n \sqrt[n]{v(\delta)}$

For any primal-feasible $\mathrm{x}$ and dual-feasible $\delta$ but this is not true of the pseudodual fuzzy modified signomial GP problem.

Corollary 2: When the values of $\sigma_{l i}$ is 1 , then a fuzzy modified signomial geometric programming (FMSGP) problem transform to ordinary modified geometric programming problem.

Theorem 2: When $\sigma_{i}$ is 1 , then $g_{0}\left(x_{i j}, s\right) \geq \mathrm{n}^{n} \sqrt{v(\delta)}$ (Primal- Dual Inequality).

Proof.

The expression for $g_{0}\left(x_{i j}, s\right)$ can be written as

$g_{0}\left(x_{i j}, s\right)=\sum_{l=1}^{n} \sum_{i=1}^{k} \delta_{i k}\left(\frac{\left(\sigma_{i i}{ }^{L}\right)^{1-s_{(}}\left(c_{l i}{ }^{U}\right)^{s} \Pi_{j=1}^{m} x_{i j}{ }^{\alpha_{i i j}}}{\delta_{i k}}\right)$.

Here the weights are $\delta_{l 1}, \delta_{l 2}, \ldots \ldots, \delta_{l k}$ and positive terms are

$\frac{\left(c_{\mathrm{ln}}\right)^{1-s_{(}}\left(c_{\mathrm{ln}}\right)^{s} \Pi_{j=1}^{m} x_{j}{ }^{\alpha_{\mathrm{ln} j}}}{\delta_{\mathrm{ln}}}$,

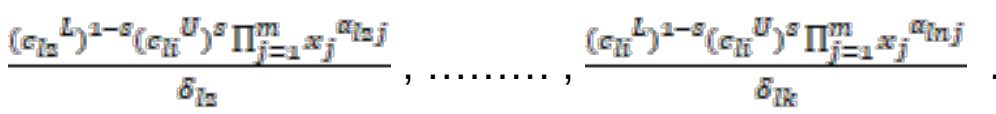

Now applying A.M.-.G.M inequality, we get

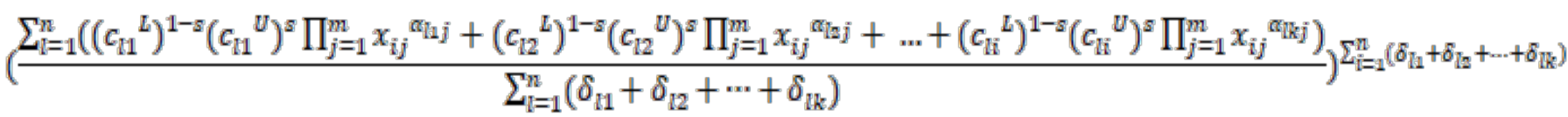

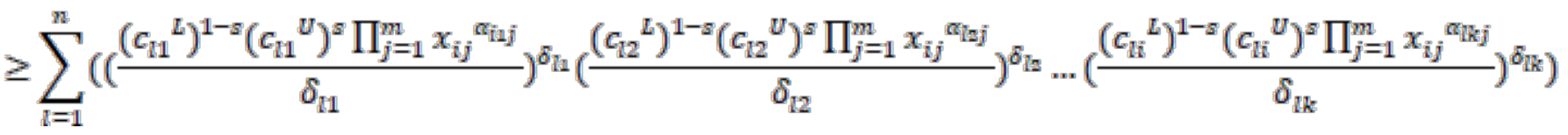

Or $\quad\left(\frac{g_{0}\left(x_{i j}, s\right)}{\sum_{l=1}^{n} \sum_{i=1}^{k} \delta_{l i}}\right)^{\sum_{l=1}^{n} \sum_{i=1}^{k} \delta_{l i}} \geq \prod_{l=1}^{n} \prod_{i=1}^{k}\left(\frac{\left(c_{l i}\right)^{1-s_{i}}\left(c_{l i}\right)^{U}{ }^{s} \Pi_{j=1}^{m} x_{i j}{ }^{\alpha_{l i j}}}{\delta_{i k}}\right)^{\delta_{i k}}$

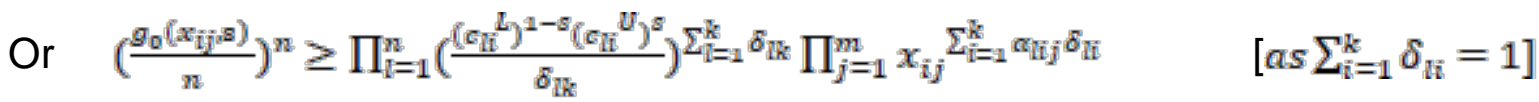

$=\prod_{i=1}^{n} \prod_{i=1}^{k}\left(\frac{\left(c_{l i}{ }^{L}\right)^{1-s}\left(c_{l i}{ }^{U}\right)^{s}}{\delta_{l i}}\right)^{\delta_{l i}} \prod_{j=1}^{m} x_{i j} \sum_{i=1}^{k} \alpha_{l i j} \delta_{l i}$ 
ISSN: 2236-269X

DOI: 10.14807/ijmp.v8i4.640

Or

$\left(\frac{g_{0}\left(x_{i j}, s\right)}{n}\right)^{n} \geq \prod_{l=1}^{n} \prod_{i=1}^{k}\left(\frac{\left(c_{l i}^{L}\right)^{1-s_{(}}\left(c_{l i}\right)^{g}}{\delta_{l i}}\right)^{\delta_{l i}}$

$$
=v(\delta)
$$

i.e., $\quad g_{0}\left(x_{i j}, s\right) \geq \mathrm{n} \sqrt[n]{v(\delta)}$.

Ex.2: Minimize $\widehat{\operatorname{Tac}}\left(Q_{i}, S_{i}\right)=\sum_{i=1}^{n} \frac{{\widetilde{\sigma_{01}}}_{i}}{Q_{i}}+\frac{\widetilde{\sigma_{h}}\left(Q_{i}-S_{i}\right)^{2}}{2 Q_{i}}+\frac{\widetilde{\sigma_{s i}}\left(S_{i}\right)^{2}}{2 Q_{i}}$

subject to $Q_{i}, S_{i}>0$

With input values

Table: 3 (Input data)

\begin{tabular}{|c|c|c|c|c|}
\hline $\mathrm{i}$ & $\widetilde{\mathrm{c}_{01}}=\widetilde{20}$ & $\widetilde{\mathrm{c}_{\mathrm{hl}}}=\widetilde{50}$ & $\widetilde{\mathrm{c}_{\mathrm{sl}}}=\widetilde{50}$ & $\mathrm{D}_{\mathrm{i}}$ \\
\hline $\mathrm{i}=1$ & $(16,20,24)$ & $(40,50,60)$ & $(40,50,60)$ & 10 \\
\hline $\mathrm{i}=2$ & $(6,10,14)$ & $(105,125,145)$ & $(21,25,29)$ & 15 \\
\hline
\end{tabular}

Using nearest approximation method

$\widetilde{10}=(6,10,14) \approx[8,12] \approx 8^{1-s} 12^{s} \in[8,12] ;$

$\widetilde{20}=(16,20,24) \approx[18,22] \approx 18^{1-s} 22^{s} \in[18,22]$

$\widetilde{25}=(21,25,29) \approx[23,27] \approx 23^{1-s} 27^{s} \in[23,27]$

$\widetilde{50}=(40,50,60) \approx[45,55] \approx 45^{1-s} 55^{\approx} \in[45,55] ;$

$\widetilde{125}=(105,125,145) \approx[115,135] \approx 115^{1-s^{2}} 135^{s} \in[115,135] ; \quad s \in[0,1]$.

Then the problem is

Min. $\operatorname{Tac}(\mathrm{Q}, \mathrm{S}, \mathrm{S})=\frac{18^{1-s_{22}} \cdot 10}{Q_{1}}+\frac{45^{1-s_{55}}\left(Q_{1}-s_{1}\right)^{2}}{2 Q_{1}}+\frac{45^{1-s_{55}}\left(S_{1}\right)^{2}}{2 Q_{1}}+\frac{18^{1-s_{2}} 22^{s} \cdot 10}{Q_{2}}+\frac{45^{1-s_{55} s}\left(Q_{2}-s_{2}\right)^{2}}{2 Q_{2}}+$ $\frac{45^{1-s_{5}} 55^{s}\left(S_{2}\right)^{2}}{2 Q_{2}}$

Sub. $\quad Q_{1}, Q_{2}, S_{1}, S_{2}>0$.

i.e.,

$\operatorname{Min} \cdot \operatorname{Tac}(\mathrm{Q}, \mathrm{S}, \mathrm{S})=\frac{18^{1-s_{22} s} \cdot 10}{Q_{1}}+$

$\frac{45^{1-s_{5}} 55^{s}\left(S_{1}\right)^{2}}{Q_{1}}+\frac{45^{1-s_{5}} 55^{s} Q_{1}}{2}-\frac{45^{1-s_{55} s^{s} S_{1}}}{1}+\frac{8^{1-s^{s}} \cdot 12^{s} \cdot 15}{Q_{2}}+\frac{\left(115^{1-s_{s}} \cdot 135^{s}+23^{1-s_{2}} 27^{s}\right)\left(S_{2}\right)^{2}}{2 Q_{2}}+\frac{115^{1-s_{1}} 135^{s} Q_{2}}{2}-$ $\frac{115^{1-e_{1}} 135^{s} S_{2}}{1}$ 
DOI: 10.14807/ijmp.v8i4.640

Sub. $Q_{1}, Q_{2}, S_{1}, S_{2}>0$.

This is primal problem and corresponding dual problem is

$$
\begin{aligned}
& v(\delta, s)=
\end{aligned}
$$

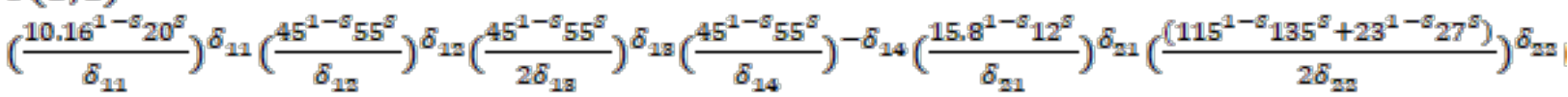

Subject to

$$
\begin{aligned}
& \delta_{11}+\delta_{12}+\delta_{13}-\delta_{14}=1, \\
& \delta_{21}+\delta_{22}+\delta_{23}-\delta_{24}=1, \\
& -\delta_{11}-\delta_{12}+\delta_{13}=0, \\
& -\delta_{21}-\delta_{22}+\delta_{23}=0, \\
& 2 \delta_{12}-\delta_{14}=0, \\
& 2 \delta_{22}-\delta_{24}=0 .
\end{aligned}
$$

Solving above equations, we have

$$
\begin{aligned}
& \delta_{14}=2 \delta_{12}, \quad \delta_{13}=1-\delta_{11}-\delta_{12}+\delta_{14}=1-\delta_{11}-\delta_{12}+2 \delta_{12}=1-\delta_{11}+\delta_{12}, \\
& \delta_{11}+\delta_{12}=1-\delta_{11}+\delta_{12} \\
& \delta_{11}=\frac{1}{2}, \delta_{13}=\frac{1}{2}+\delta_{12} .
\end{aligned}
$$

And

$$
\begin{aligned}
& \delta_{24}=2 \delta_{22}, \delta_{23}=1-\delta_{21}-\delta_{22}+\delta_{24}=1-\delta_{21}-\delta_{22}+2 \delta_{22}=1-\delta_{21}+\delta_{22} \text {, } \\
& \delta_{21}+\delta_{22}=1-\delta_{21}+\delta_{22} \\
& \delta_{21}=\frac{1}{2}, \delta_{23}=\frac{1}{2}+\delta_{22}
\end{aligned}
$$

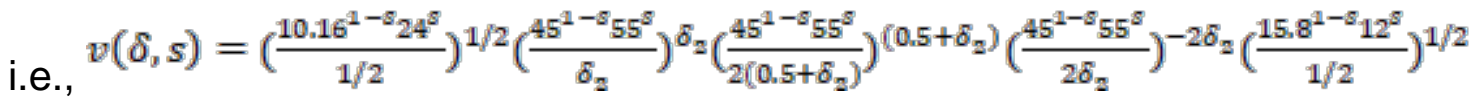

$$
\begin{aligned}
& \times\left(\frac{115^{1-s_{1}} 135^{s}+23^{1-s_{2}} 27^{s}}{2 \delta_{2}}\right)^{\delta_{2}}\left(\frac{115^{1-s_{1}} 135^{s}}{2\left(0.5+\delta_{2}\right)}\right)^{\left(0.5+\delta_{2}\right)}\left(\frac{115^{1-s_{1}} 135^{s}}{2 \delta_{2}}\right)^{-2 \delta_{2}}
\end{aligned}
$$

Taking log on both side of (6.6) and then partially differentiating with respect to $\delta_{12}$ and $\delta_{22}$ respectively and using the conditions of finding optimal solution we get; 
ISSN: 2236-269X

DOI: 10.14807/ijmp.v8i4.640

$\left(\log 2.45^{1-s} 55^{s}-\log 2 \delta_{12}\right)-0.5+\left(\log 45^{1-s} 55^{s}-\log 2\left(0.5+\delta_{12}\right)-0.5\right.$

$$
-2\left(\log 45^{1-s} 55^{s}-\log 2 \delta_{12}\right)+1=0
$$

$\Rightarrow \log \left(2.45^{1-s} 55^{s}\right) / 45^{1-s} 55^{s}-\log 2 \delta_{12}\left(1+2 \delta_{12}\right)=0$

$\Rightarrow \delta_{12}\left(1+2 \delta_{12}\right)=1$.

And

$\left(\log 2.45^{1-s} 55^{s}-\log 2 \delta_{22}\right)-0.5+\left(\log 45^{1-s} 55^{s}-\log 2\left(0.5+\delta_{22}\right)-0.5\right.$

$$
-2\left(\log 45^{1-s} 55^{s}-\log 2 \delta_{22}\right)+1=0
$$

$\Rightarrow \log \left(2.45^{1-s} 55^{s}\right) / 45^{1-s} 55^{s}-\log 2 \delta_{22}\left(1+2 \delta_{22}\right)=0$

$\Rightarrow \delta_{22}\left(1+2 \delta_{22}\right)=1$.

From primal-dual relation

$$
\begin{aligned}
& \frac{10,16^{1-s_{24}}}{Q_{1}}=\delta_{11} v(\delta), \\
& \frac{45^{1-s_{55}} s^{s} s_{1}^{2}}{Q_{1}}=\delta_{12} \sqrt{v(\delta, s)}, \\
& \frac{45^{1-s_{55}} Q_{1}}{2}=\delta_{13} \sqrt{v(\delta, s)}, \\
& 45^{1-s} 55^{s} S_{1}=\delta_{14} \sqrt{v(\delta, s)}, \\
& \frac{15,8^{1-s_{12}}{ }^{s}}{Q_{2}}=\delta_{21} \sqrt{v(\delta, s)}, \\
& \frac{\left(115^{1-s^{13}} 135^{s}+23^{1-s_{2}} 27^{s}\right) S_{2}{ }^{2}}{2 Q_{2}}=\delta_{22} \sqrt{v(\delta, s)}, \\
& \frac{115^{1-s_{13}} 5^{s} Q_{2}}{2}=\delta_{23} \sqrt{v(\delta, s)}, \\
& 115^{1-s} 135^{s} S_{2}=\delta_{24} \sqrt{v(\delta, s)} \text {. }
\end{aligned}
$$

Solving above relations with difference values of weight we get the list of values in table-4. 
INDEPENDENT JOURNAL OF MANAGEMENT \& PRODUCTION (IJM\&P)

http://www.ijmp.jor.br

v. 8, n. 4, October - December 2017

ISSN: 2236-269X

DOI: 10.14807/ijmp.v8i4.640

Table 4: optimal solution

\begin{tabular}{|c|c|c|c|c|c|}
\hline & & & & $\begin{array}{l}\text { Optimal val } \\
\text { objectives }\end{array}$ & \\
\hline $\mathrm{s}$ & $1-s$ & Optimal dual variables & $\begin{array}{l}\text { Optimal primal } \\
\text { variables }\end{array}$ & $v(\delta, s)$ & $\operatorname{Tac}(\mathrm{Q} S)$ \\
\hline 0.1 & 0.9 & $\begin{array}{l}\delta_{11}^{*}=0.5, \\
\delta_{12}^{*}=0.5, \\
\delta_{13}^{*}=1, \delta_{14}^{*}=1, \\
\delta_{21}^{*}=0.5 \\
\delta_{22}^{*}=0.5 \\
\delta_{23}^{*}=1, \delta_{24}^{*}=1 .\end{array}$ & $\begin{array}{l}\mathrm{S}_{1}^{*}=1.971, \\
\mathrm{Q}_{1}^{*}=3.912, \\
\mathrm{~S}_{2}^{*}=0.774, \\
\mathrm{Q}_{2}^{*}=1.549 .\end{array}$ & 8187.095 & 195.347 \\
\hline 0.3 & 0.7 & $\begin{array}{l}\delta_{11}^{*}=0.5, \\
\delta_{12}^{*}=0.5, \\
\delta_{13}^{*}=1, \delta_{14}^{*}=1, \\
\delta_{21}^{*}=0.5, \\
\delta_{22}^{*}=0.5, \\
\delta_{23}^{*}=1, \delta_{24}^{*}=1 .\end{array}$ & $\begin{array}{l}\mathrm{S}_{1}^{*}=2.008, \\
\mathrm{Q}_{1}^{*}=4.015 \\
\mathrm{~S}_{2}^{*}=0.795, \\
\mathrm{Q}_{2}^{*}=1.590 .\end{array}$ & 9205.062 & 206.989 \\
\hline 0.5 & 0.5 & $\begin{array}{l}\delta_{11}^{*}=0.5, \\
\delta_{12}^{*}=0.5, \\
\delta_{13}^{*}=1, \delta_{14}^{*}=1, \\
\delta_{21}^{*}=0.5 \\
\delta_{22}^{*}=0.5 \\
\delta_{23}^{*}=1, \delta_{24}^{*}=1 .\end{array}$ & $\begin{array}{l}\mathrm{S}_{1}^{*}=2.045, \\
\mathrm{Q}_{1}^{*}=4.090, \\
\mathrm{~S}_{2}^{*}=0.816, \\
\mathrm{Q}_{2}^{*}=1.633 .\end{array}$ & 10349.600 & 219.326 \\
\hline 0.7 & 0.3 & $\begin{array}{l}\delta_{11}{ }^{*}=0.5, \\
\delta_{12}{ }^{*}=0.5, \\
\delta_{13}{ }^{*}=1, \delta_{14}^{*}=1, \\
\delta_{21}^{*}=0.5, \\
\delta_{22}{ }^{*}=0.5, \\
\delta_{23}{ }^{*}=1, \delta_{24}^{*}=1 .\end{array}$ & $\begin{array}{l}\mathrm{S}_{1}^{*}=2.083, \\
\mathrm{Q}_{1}^{*}=4.166, \\
\mathrm{~S}_{2}^{*}=0.838, \\
\mathrm{Q}_{2}^{*}=1.677 .\end{array}$ & 11636.450 & 232.372 \\
\hline 0.9 & 0.1 & $\begin{array}{l}\delta_{11}^{*}=0.5, \\
\delta_{12}^{*}=0.5, \\
\delta_{13}^{*}=1, \delta_{14}^{*}=1, \\
\delta_{21}^{*}=0.5 \\
\delta_{22}^{*}=0.5 \\
\delta_{23}^{*}=1, \delta_{24}^{*}=1 .\end{array}$ & $\begin{array}{l}\mathrm{S}_{1}^{*}=2.122, \\
\mathrm{Q}_{1}^{*}=4.244, \\
\mathrm{~S}_{2}^{*}=0.861, \\
\mathrm{Q}_{2}^{*}=1.722 .\end{array}$ & 13083.300 & 246.191 \\
\hline
\end{tabular}

\section{CONCLUSION}

In this paper, a fuzzy EOQ model with shortages under fully backlogging and constant demand is formulated and solved. Here the model is solved by fuzzy signomial geometric programming (FSGP) technique. For fuzzy coefficient we used only triangular fuzzy number (TrFN). In future other types of fuzzy numbers would be used. The methodology proposed in this paper may also be applicable to other EOQ models. 
Our approach provide here a simple EOQ model, but in the future it should be used many complex EOQ models. For future research of uncertainty in economic order quantity (EOQ) model, by using different type of fuzzy numbers such as pentagonal, hexagonal fuzzy numbers of generalized fuzzy numbers be analytically more challenging and interesting. Inflation plays an important role in present day-today life, but we have neglected it. Therefore, consideration of inflation problem would be more realistic.

\section{REFERENCE:}

APPADOO, S. S.; BECTOR, C. R.; BHATT, S. K. (2012) Fuzzy EOQ model using possibilistic approach, Journal of Advances in Management Research, v. 9 n. 1, p.139-164.

BELLMAN, R. E.; ZADEH (1970). Decision making in a fuzzy environment, Management Science, v. 17, p. B141-B164.

BLAU, G.; WILDE, D. J. (1967) Second Order Characterization of Generalized Polynomial Programs, Princeton International Symp. Math. Programming.

CARLSSON, C.; FULLER, R. (2001) On Possibilistic Mean Value and Variance of Fuzzy Numbers. Fuzzy Sets and Systems, n. 122, p. 315-326.

CARLSSON, C.; FULLER, R. (2002) Fuzzy Reasoning in Decision Making and Optimization, Physics-Verlag.

CHARNES, A.; COPPER, W. W.; GOLANY, B.; MASRERS, J. (1988) Optimal dwsine modification by geometric programming and constrained stochastic network models. International Journal of System Science, v. 19 p. 825-844.

CLARK, A. J. (1992). An informal survey of multy-echelon inventory theory, Naval Research Logistics Quarterly, n. 19, p. 621-650.

DUTTA, D.; KUMAR, P. (2012). Fuzzy inventory without shortages using trapezoidal fuzzy number with sensitivity analysis, IOSR Journal of Mathematics, v. 4, n. 3, p. 32-37.

DUTTA, D.; RAO, J. R.; TIWARY R. N. (1993) Effect of tolerance in fuzzy linear fractional programming, Fuzzy Sets and Systems, n. 55, p. 133-142.

HAMACHER, LEBERLING, H.; ZIMMERMANN, H. J. (1978). Sensitivity Analysis in fuzzy linear Programming Fuzzy Sets and Systems, n. 1, p. 269-281

HARRIS, FORD W. (1990) How Many Parts to Make at Once. Operations Research, v. 8, n. 6, p. 947.

ISLAM, S.; ROY, T. K. (2006). A fuzzy EPQ model with flexibility and reliability consideration and demand depended unit Production cost under a space constraint: A fuzzy geometric programming approach, Applied Mathematics and Computation, v. 176, n. 2, p. 531-544.

ISLAM, S.; ROY, T. K. (2010). Multi-Objective Geometric-Programming Problem and its Application. Yugoslav Journal of Operations Research, n. 20, p. 213-227. 
KOTB A. M.; HLAA. A.; FERGANCY (2011). Multi-item EOQ model with both demand-depended unit costand varying Leadtime via Geometric Programming, Applied Mathematics, n. 2, p. 551-555.

KHUN, H. W.; TUCKER A. W. (1951). Non-linear programming, proceeding second Berkeley symposium Mathematical Statistic and probability (ed) NYMAN, J. University of California press, p. 481-492.

LIANG, Y.; ZHOU, F. (2011), A two warehouse inventory model for deteriorating items under conditionally permissible delay in Payment, Applied Mathematical Modeling, n. 35, p. 2221-2231.

LIU, S. T. (2006). Posynomial Geometric-Programming with interval exponents and co-efficients, Europian Journal of Operations Research, v. 168, n. 2, p. 345-353

MAITY, M. K. (2008). Fuzzy inventory model with two ware house under possibility measure in fuzzy goal, European Journal Operation Research, n. 188, p.746-774

MANDAL, W. A.; ISLAM, S. (2016). Fuzzy Inventory Model for Deteriorating Items, with Time Depended Demand, Shortages, and Fully Backlogging, Pak.. j. stat. oper.res., v. XII, n.1, p.101-109.

MANDAL, W. A.; ISLAM, S. (2015). Fuzzy Inventory Model for Power Demand Pattern with Shortages, Inflation Under Permissible Delay in Payment, International Journal of Inventive Engineering and Sciences, v. 3, n. 8.

MANDAL, W. A.; ISLAM, S. (2015). Fuzzy Inventory Model for Weibull Deteriorating Items, with Time Depended Demand, Shortages, and Partially Backlogging, International Journal of Engineering and Advanced Technology, v. 4, n. 5.

MANDAL, W. A.; ISLAM, S. (2016). A Fuzzy E.O.Q Model with Cost of Interest, Time Depended Holding Cost, With-out Shortages under a Space Constraint: A Fuzzy Geometric Programming and Non-Linear Programming Approach. International Journal of Research on Social and Natural Sciences, v. I, n. 1, June, p. 134147.

PASSY, U.; WILDE, D. J. (1967) Generalized Polynomial Optimization, SIAM Jour. Appli. Math., n. 15, p. 1344-1356.

PASSY, U.; WILDE, D. J. (1968) A Geometric Programming Algorithm for Solving Chemical Equilibrium Problems, SIAM Jour. Appli. Math., n. 16, p. 363-373.

ROY, T. K.; MAYTY, M. (1995). A fuzzy inventory model with constraints, Opsearch, v. 32, n. 4, p. 287-298.

ZADEH, L. A. (1965), Fuzzy sets, Information and Control, n. 8, p. 338-353.

ZIMMERMANN, H. J. (1985) Application of fuzzy set theory to mathematical programming, Information Science, n. 36, p. 29-58.

ZIMMERMANN, H. J. (1992) Methods and applications of Fuzzy Mathematical programming, In: YAGER, R. R.; ADEH, L. A. Z. (eds), An introduction to Fuzzy Logic Application in Intelligent Systems, p.97-120, Kluwer publishers, Boston. 